\title{
Analysis of the evolution game of stakeholders' behaviour in the operation stage of green buildings
}

\author{
Xiaojun Liu and Xiaodan Liu* \\ School of Management Xi' an University of Architecture and Technology, Xi'an 710055, China
}

This study aims to solve the problem of uncoordinated interests among the stakeholders in the operation stage (OS) of green buildings (GBs). First, the requirements and conflicts between the owners and property service enterprises (PSEs) are analysed. Secondly, the evolutionary game model of owners and PSEs is developed. It was found that the game system will evolve towards the ideal equilibrium stability strategy only under the following conditions: (i) the income of owners in green property management (GPM) is greater than the sum of the income of PSEs shared by the owner in GPM and the transaction costs generated when the owners choose to support GPM, and (ii) the sum of the income of PSEs shared by the owner in GPM and the additional benefits that PSEs can get by choosing GPM is greater than the total cost incurred by PSEs in choosing GPM. We carried out quantitative tests for equilibrium stability results and analysed the factor that influence the evolution path based on simulations performed in MATLAB. The results show that improving the operational income of GBs, increasing the additional income of PSEs, reducing the operating transaction costs and adopting a reasonable revenue sharing method are effective ways to coordinate the interests of all parties involved in the OS of GBs.

Keywords: Evolutionary game theory, green buildings, operation stage, stakeholders.

AT present, China is facing the very serious task of energy conservation and emission reduction. Green buildings (GBs) can effectively reduce energy consumption and reduce greenhouse gas emissions ${ }^{1,2}$. GBs involve multiple stages such as design, construction and operation ${ }^{3}$, among which the operation stage (OS) is the most important in the whole life cycle of $\mathrm{GBs}^{4}$, more than $80 \%$ of energy consumption occurs during the actual $\mathrm{OS}^{5}$. Due to lack of effective operation management, a large number of practical operation effects of GBs has not achieved the desired goal $^{6}$. The OS of GBs involves a number of stakeholders, such as the government, owners and end-users, and a

*For correspondence. (e-mail: liu_xiao_dan_91@163.com) variety of objectives, including social, environmental and economic interests ${ }^{7}$. These objectives are not always compatible among stakeholders, thus giving rise to conflicts which seriously impede the effective operation of $\mathrm{GBs}^{8}$. Therefore, it is necessary to clarify the interest relation and coordinate their conflict of interest among stakeholders in the OS of GB for realizing the energy saving effect of GB.

Game theory is the main tool to analyse the multi-agent behaviour selection strategy and solve problems of conflict or cooperation between decision-makers ${ }^{9}$. So a lot of literatures have analysed the behaviour strategy of each subject and their interest conflict in the diffusing GB. Among them, the gaming relationships between the government and developers ${ }^{10}$, developers and consumers ${ }^{11}$, government, developers and consumers ${ }^{12}$, and government and owners ${ }^{13}$ are the key topics. With the increasingly prominent problem of GBs in OS, the importance of property service enterprises (PSEs) cannot be ignore $^{14}$. Therefore, the game relationship between government and PSEs was examined ${ }^{15}$. However, the existing literature still lacks analysis of the game behaviour between PSEs and owners. Moreover, the study about the verification of equilibrium stable results and simulation of system evolution paths for different influence factors is not enough. Therefore, this study aims to analyse the game stability strategies between owners and PSEs, and identify the factors that influence the stability strategies.

\section{Requirements and conflicts among stakeholders}

\section{Requirements}

PSEs hope to maximize their own economic benefits, and to obtain stable or additional economic benefits through daily operations of the buildings. The energy-saving demand brings new profit opportunities to PSEs. However, it is not common for PSEs to offer green property management (GPM). This is because GPM needs active cooperation with the owners. Also, the operational management cost of GBs is higher than traditional buildings, thus most of PSEs consider GBs as a burden, and discourage the use of green technologies. 
In the OS of GBs, the owners pay more attention to the operation and maintenance costs and the economic benefits generated by energy-saving. Also, the owners tend to focus on their own interests and are not willing to pay more while obtaining economic benefits in the process of operational management, which often leads to conflict of interest between the PSEs and owners.

\section{Conflicts}

Traditional property management (TPM) is difficult to meet the operational requirements of GBs. GPM can achieve resource conservation, but also increases the operational and management costs. PSEs cannot directly share the energy-saving profits, which means their interest requirements are not met, leading to PSEs becoming reluctant to spend money on promoting green operating ability. For the owners, it is not clear regarding the energysaving benefits. And the lack of green operation capacity will actually cause the owners' losses when the information is asymmetrical. Therefore, the inequality between green operating cost and energy-saving benefits leads to interest conflicts between the owners and PSEs.

\section{Evolutionary game of owners and PSEs}

\section{Basic assumption}

There are two limited rationality agents, namely owners and PSEs. At the beginning, it is not possible for these agents to directly select the strategy that is beneficial to them. A stable state of evolution can be reached in the process of strategy adjustment by mutual imitation and learning. At time $t$, let the proportion of those 'supporting GPM' and 'not supporting GPM' in the group of owners be $x(t)$ and $1-x(t)$ respectively. Let ratio of those 'providing GPM' and 'providing TPM' in the PSEs group be $y(t)$ and $1-y(t)$ respectively. In the following, $t$ is omitted from $x(t)$ and $y(t)$ for ease notation. Based on the above assumptions, the payoff matrix and variable description are shown in Tables 1 and 2 respectively.

\section{Evolutionary stabilization strategy of owners}

For owners, the expected benefits of choosing to support GPM are $E_{A 1}$; otherwise it is $E_{A 2}$. The average expected benefits are represented as $E_{A}$ :

$$
\begin{aligned}
E_{A 1} & =y\left(a_{1}+e-w-h\right)+(1-y)\left(a_{1}-h\right) \\
& =y(e-w)+a_{1}-h, \\
E_{A 2} & =y a_{1}+(1-y) a_{1}=a_{1},
\end{aligned}
$$

$$
E_{A}=x E_{A 1}+(1-x) E_{A 2}=x y(e-w)-x h+a_{1} .
$$

The replicator dynamics equation of the owner is

$$
\begin{aligned}
F(x) & =\frac{\mathrm{d} x}{\mathrm{~d} t}=x\left(E_{A 1}-E_{A}\right) \\
& =x(1-x)\left(E_{A 1}-E_{A 2}\right)=x(1-x)[y(e-w)-h] .
\end{aligned}
$$

Order $F(x)=\mathrm{d} x / \mathrm{d} t=0$, then $x_{1}=0, x_{2}=1, y^{*}=h /(e-w)$, the condition of the evolutionary stabilization strategy (ESS) of $x$ is $F(x)=0, F^{\prime}(x)<0$. Thus, three stable states of the owners can be obtained:

(i) When $y=y^{*}=h /(e-w), F(x)=0, F^{\prime}(x)=0$, all values of $x$ are in a stable state, which means that ESS of the owners is random when the proportion of choosing GPM for PSEs in the condition of $y=y^{*}=h /(e-w)$.

(ii) When $y<y^{*}<h /(e-w)$ there may only be two steady-state points, namely $x_{1}=0, x_{2}=1$. For $F(0)=0$, $F^{\prime}(0)>0, F(1)=0$ and $F^{\prime}(1)<0, x_{1}=1$ is the owner's ESS. This indicates that the owner who chooses not to support GPM will gradually become an ESS when the proportion for PSE to choose the GPM is $y<(e-w)$.

(iii) When $y>y^{*}$, there are only two steady-state points, namely $x_{1}=0, x_{2}=1$. For $F(0)=0, F^{\prime}(0)>0, F(1)=0$ and $F^{\prime}(1)<0, x_{1}=1$ is the owner's ESS. This indicates that the owner who chooses to support GPM will gradually become an ESS when the proportion for PSE to choose the GPM in the condition of $y>y^{*}$.

\section{Evolutionary stabilization strategy of PSEs}

For PSEs, the expected benefits of choosing to support GPM are $E_{B 1}$, otherwise it is $E_{B 2}$. The average expected benefits are represented as $E_{B}$.

Table 1. Pay-off matrix of property service enterprises (PSEs) and owners

\begin{tabular}{lcc}
\hline & \multicolumn{2}{c}{ PSE } \\
\cline { 2 - 3 } Owner & $\begin{array}{c}\text { Green property } \\
\text { management }(\mathrm{GPM})\end{array}$ & $\begin{array}{c}\text { Traditional property } \\
\text { management (TPM) }\end{array}$ \\
\hline Supporting & $a_{1}+e-w-h, a_{2}+w+r-c$ & $a_{1}-h, a_{2}$ \\
Not supporting & $a_{1}, a_{2}-c$ & $a_{1}, a_{2}$ \\
\hline
\end{tabular}

Table 2. Description of variables

\begin{tabular}{ll}
\hline Variable & \multicolumn{1}{c}{ Description } \\
\hline$a_{1}$ & Income of owners in TPM \\
$a_{2}$ & Income of PSEs in TPM \\
$e$ & Income of owners in GPM \\
$w$ & Income of PSEs shared by the owner in GPM \\
$h$ & Transaction costs generated when owners choose to support \\
& GPM \\
$c$ & Total cost incurred by PSEs in choosing GPM \\
$r$ & Additional benefits that PSEs can get by choosing GPM \\
\hline
\end{tabular}




$$
\begin{aligned}
E_{B 1}= & x\left(a_{2}+w+r-c\right) \\
& +(1-x)\left(a_{2}-c\right)=x(w+r)+a_{2}-c, \\
E_{B 2}= & x a_{2}+(1-x) a_{2}=a_{2}, \\
E_{B}= & y E_{B 1}+(1-y) E_{B 2}=y x(w+r)-y c+a_{2} .
\end{aligned}
$$

The replicator dynamics equation of PSEs is

$$
\begin{aligned}
F(y) & =\frac{\mathrm{d} y}{\mathrm{~d} t}=y\left(E_{B 1}-E_{B}\right) \\
& =y(1-y)\left(E_{B 1}-E_{B 2}\right)=y(1-y)[x(w+r)-c] .
\end{aligned}
$$

Order $F(y)=\mathrm{d} y / \mathrm{d} t=0$, then $y_{1}=0, \quad y_{2}=1, \quad x^{*}=c /$ $(w+r)$, the condition of ESS of $y$ is $F(y)=0, F^{\prime}(y)<0$. Thus, three stable states of the PSEs can be obtained.

(i) When $x=x^{*}=c /(w+r), F(y)=0, F^{\prime}(y)=0$, all values of $y$ are in a stable state, which means the ESS of PSEs is random when the proportion for owners to choose the GPM is $x=x *$.

(ii) When $x<x^{*}$, there are only two steady state points, namely $y_{1}=0, y_{2}=1$. For $F(0)=0, F^{\prime}(0)<0, F(1)=0$ and $F^{\prime}(1)>0, y_{1}=0$ is the PSE's ESS, which means the PSE who chooses to support TPM will gradually become an ESS when the proportion for owners to choose the GPM is $\mathrm{x}<x^{*}$.

(iii) When $x>x^{*}$, there are only two steady state points, namely $y_{1}=0, y_{2}=1$. For $F(0)=0, F^{\prime}(0)>0$, $F(1)=0$ and $F^{\prime}(1)<0, y_{1}=1$ is the PSE's ESS, which means the owner who chooses to support GPM will gradually become an ESS when the proportion for owner to choose the GPM is $x>x^{*}$.

\section{Analysis of equilibrium stability of the game system}

Five local equilibrium points, namely $O(0,0), A(0,1), B$ $(1,0), C(1,1), D(c /(w+r), h /(e-w))$, can be obtained by solving eq. (9) below

$$
\left\{\begin{array}{l}
F(x)=x(1-x)[y(e-w)-h]=0, \\
F(y)=y(1-y)[x(w+r)-c]=0 .
\end{array}\right.
$$

Taking the partial derivative of $F(x)$ and $F(y)$ with respect to $x$ and $y$, we can obtain the Jacobian matrix $(J)$. The five local equilibrium points of the game system are substituted into $J$, and their local stability can be determined through the sign of their determinant $(\operatorname{det}(J))$ and trace $(\operatorname{tr}(J))$ values. This equilibrium point is ESS if and only if $\operatorname{det}(J)>0$ and $\operatorname{tr}(J)<0$. As shown in Table 3, $\operatorname{det}(J)$ and $\operatorname{tr}(J)$ of each local equilibrium point are calculated.

$$
\begin{gathered}
J=\left[\begin{array}{cc}
(1-2 x)[y(e-w)-h] & x(1-x)(e-w) \\
y(1-y)(w+r) & (1-2 y)[x(w+r)-c]
\end{array}\right], \\
\operatorname{det}(J)=(1-2 x)[y(e-w)-h](1-2 y)[x(w+r)-c] \\
-x(1-x)(e-w) y(1-y)(w+r), \\
\operatorname{tr}(J)=(1-2 x)[y(e-w)-h]+(1-2 y)[x(w+r)-c] .
\end{gathered}
$$

In order to judge the stability of the five equilibrium points, it is necessary to discuss the positive and negative conditions of their $\operatorname{det}(J)$ and $\operatorname{tr}(J) . D(c /(w+r), h /(e-w))$ is nonexistent in three cases namely (i) $e<w+h$, $w+r<c$, (ii) $e>w+h, w+r<c$ and (iii) $e<w+h$, $w+r>c$. Table 4 shows the evolutionary stability result of $O(0,0), A(0,1), B(1,0), C(1,1)$, while Figure 1 shows the evolutionary phase diagram. Since the game system has only one stable point, viz. $O(0,0)$, the evolutionary equilibrium result of any point in the phase diagram will tend to the stable point $(0,0)$. For the condition $e>w+h, w+r>c$, the evolutionary stability result of $O(0,0), A(0,1), B(1,0), C(1,1)$ and $D(c /(w+r)$, $h /(e-w))$ are shown in Table 5, while Figure 2 shows the evolutionary phase diagram. Since the game system has only two stable points, viz. $O(0,0)$ and $C(1,1)$, the evolutionary equilibrium result of any point in the phase diagram will tend to the stable point $(0,0)$ or $(1,1)$.

The strategy of the owners and PSEs may evolve to the ideal equilibrium point $C(1,1)$ only in case 3 , which needs to be discussed in detail. Figure 3 shows the dynamic evolution process of the game strategy between the owners and PSEs. When $x$ and $y$ are in the shadow area, the game system will gradually evolve to the ideal equilibrium point $C(1,1)$. When $x$ and $y$ are in the blank area, the game system will gradually evolve to the ideal equilibrium point $O(0,0)$. Thus, the equilibrium result of the system is determined by the position of $(x, y)$, and the path will evolve to which state depending on the saddle point $D(c /(w+r), h /(e-w))$.

\section{Analysis of equilibrium stability test and the factor that influence the evolution path based on numerical simulation}

\section{Equilibrium stability test}

The evolution path is presented in two-dimensional plane coordinates, the horizontal axis is time $t$. The initial time and the end time is set to 0 and 1 respectively. The vertical axis is $x$ and $y$, and their values range from 0 to 1 . The initial state is randomly taken in the range $(x, y)$ for the sake of generality. Testing the equilibrium results under the conditions: (i) $e<w+h, w+r<c$ (taking $e=4, w=3$, $h=2, \quad c=6, r=2)$; (ii) $e<w+h, w+r>c \quad(e=4$, 

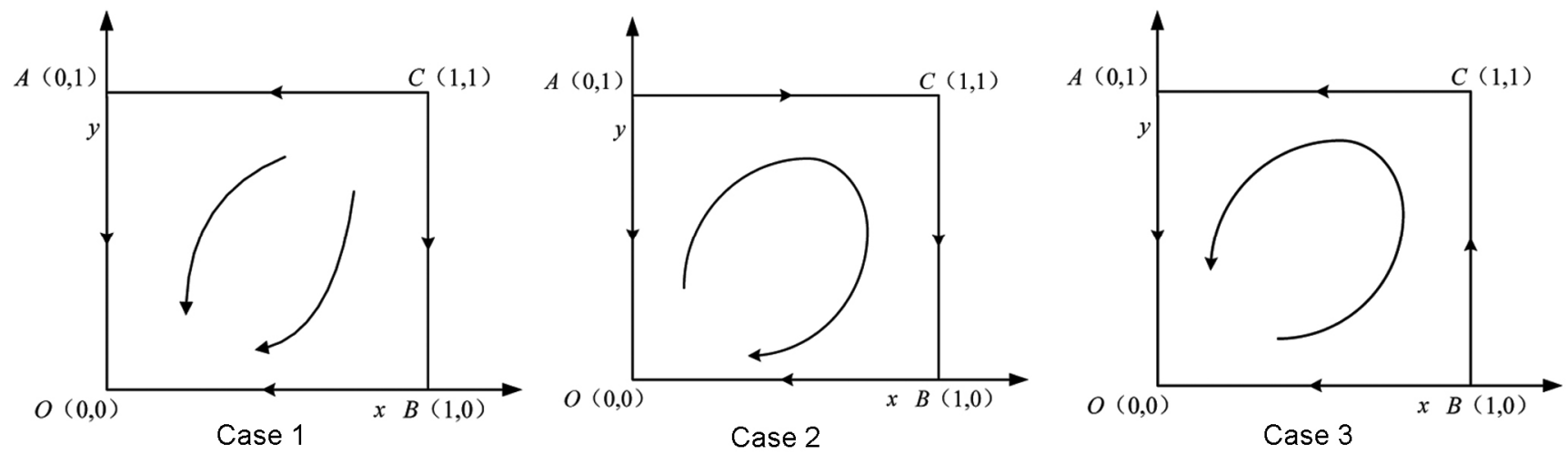

Figure 1. The evolutionary phase diagram of cases 1-3.

Table 3. Results of partial equilibrium of $\operatorname{det}(J)$ and $\operatorname{tr}(J)$

\begin{tabular}{lcc}
\hline Local equilibrium point & $\operatorname{det}(J)$ & $\operatorname{tr}(J)$ \\
\hline$O(0,0)$ & $h c$ & $-h-c$ \\
$A(0,1)$ & $c(e-w-h)$ & $c+e-w-h$ \\
$B(1,0)$ & $h(w+r-c)$ & $h+c-e-r$ \\
$C(1,1)$ & $(e-w-h)(w+r-c)$ & $h+c-e-r$ \\
$D\left(\frac{c}{w+r}, \frac{h}{e-w}\right)$ & $\frac{h c(w+r-c)(e-w-h)(e-w)(w+r)}{(w+r)^{2}(e+w)^{2}}$ & 0 \\
\hline
\end{tabular}

Table 4. Evolutionary stable result of cases 1-3

\begin{tabular}{|c|c|c|c|c|c|c|c|c|c|}
\hline \multirow[b]{2}{*}{ Equilibrium point } & \multicolumn{3}{|c|}{ Case 1} & \multicolumn{3}{|c|}{ Case 2} & \multicolumn{3}{|c|}{ Case 3} \\
\hline & $\operatorname{det}(J)$ & $\operatorname{tr}(J)$ & Stability & $\operatorname{det}(J)$ & $\operatorname{tr}(J)$ & Stability & $\operatorname{det}(J)$ & $\operatorname{tr}(J)$ & Stability \\
\hline$O(0,0)$ & + & - & Stable & + & - & Stable & + & - & Stable \\
\hline$A(0,1)$ & - & Uncertainly & Saddle point & + & + & Unstable & - & Uncertainly & Saddle point \\
\hline$B(1,0)$ & + & Uncertainly & Saddle point & - & Unstable & Saddle point & + & + & Unstable \\
\hline$C(1,1)$ & + & + & Unstable & - & Unstable & Saddle point & - & Uncertainly & Saddle point \\
\hline
\end{tabular}

Table 5. Stability of the local equilibrium point when $e>w+h$,

\begin{tabular}{lccc}
\multicolumn{4}{c}{$w+r>c$} \\
\hline Local equilibrium point & $\operatorname{det}(J)$ & $\operatorname{tr}(J)$ & Stability \\
\hline$O(0,0)$ & + & - & Stable \\
$A(0,0)$ & + & + & Unstable \\
$B(0,0)$ & + & + & Unstable \\
$C(0,0)$ & + & - & Stable \\
$D\left(\frac{c}{w+r}, \frac{h}{e-w}\right)$ & + & 0 & Saddle point \\
\hline
\end{tabular}

$w=3, h=2, c=4, r=2)$; (iii) $e>w+h, w+r<c(e=6$, $w=3, h=2, \quad c=6, \quad r=2$ ); (iv) $e>w+h, \quad w+r>c$ $(e=6, w=3, h=2, c=4, r=2)$. Figure 4 shows the evolutionary path. It can be seen that the system equilibrium result eventually evolves to a stable point in every condition, which is consistent with theoretical results.

\section{Influencing factor of evolution path}

The dynamic influence of $e, w, h, c$ and $r$ on the evolution path of the system is simulated by changing the initial value. Assume that the initial values of each parameter are $e=8, w=4, h=2, c=3, r=1$, and these values satisfy the inequality $e>w+h, w+r>c$. Taking the initial state of evolution $t=0$, then $x(0)=0.62, y(0)=0.51$, the initial state is close to the saddle point and tends to evolve to the position of the ideal equilibrium point $C(1$, 1). Figure 5 shows the evolution path of the game system under initial conditions. We can see that the system will evolve towards an ideal state for operational management of GBs as the time $t$ changes.

\section{Impact of operating income on evolution path}

As shown in Figure 6, when $e$ increases to 10, as the time $t$ changes, the proportion of the owners supporting GPM 
tends to 1 at a faster rate. However, when $e$ reduces to 7 , as $t$ changes, the proportion of the owners supporting GPM is gradually increased from 0.62 to 0 , and the proportion of PSEs choosing to carry out the GPM is gradually increased from 0.51 to 0 . This means that the system will evolve towards an ideal state and unsatisfactory state that is not conducive to operational management of GBs respectively.

\section{Impact of revenue sharing amount on evolution path}

As shown in Figure 7, when $w$ decreases to 3, as the time $t$ changes, the proportion of owners supporting GPM gradually increases, and then tends to 0 with the proportion of PSEs choose GPM tending to 0 . When $w$ increases to 5 , as the time $t$ changes, the proportion of PSEs supporting GPM gradually increases, and then tends to 0 with the proportion of owners choosing GPM tending to 0 . These indicate that the system will evolve to a state that is not conducive to operational management of GBs.

\section{Impact of owner's transaction costs on evolution path}

As shown in Figure 8, when $h$ increases to 3, as the time $t$ changes, the system will evolve towards a state that

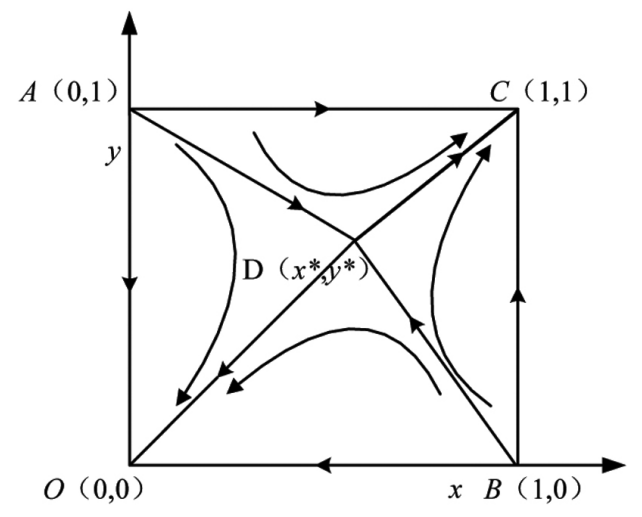

Figure 2. Evolutionary phase diagram when $e>w+h, w+r>c$.

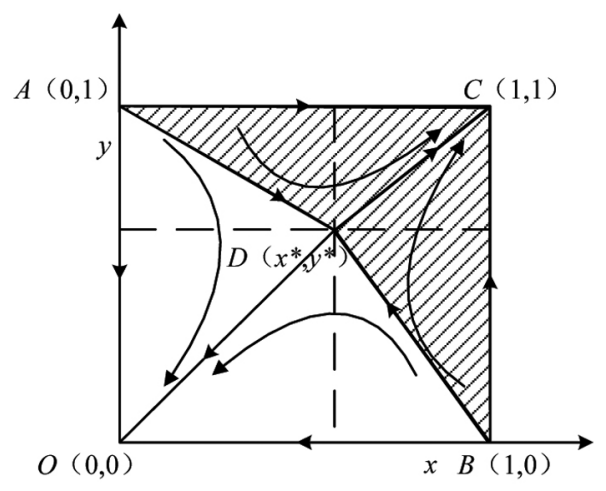

Figure 3. Evolutionary phase diagram of the game system. is not conducive to operational management of GBs. However, when $h$ decreases to 1 , as $t$ changes, the proportion of owners supporting green operations tends to 1 at a faster rate, and the system will evolve towards an ideal state for operational management of GBs.

\section{Impact of operating cost of PSEs on evolution path}

As shown in Figure 9, when the operating cost of PSEs takes $c=3$ at the initial condition, as the time $t$ changes, the proportion of owners to supporting green operations gradually increases from 0.62 to 1 , and the ratio of PSEs choosing to carry out green operations gradually increases from 0.51 to 1 , indicating that the system will evolve towards an ideal state for operational management of GBs. Taking the initial conditions as a reference, when the operating cost of PSEs increases to 4 , as $t$ changes, the system will evolve towards a state that is not conducive to operational management of GBs. However, when $c$ decreases to 2 , as $t$ changes, the proportion of PSEs supporting green operations tends to 1 at a faster rate, and the system will evolve toward an ideal state for operational management of GBs.

\section{Impact of extra income of PSEs on evolution path}

As shown in Figure 10, when $r$ increases to 2, as the time $t$ changes, the proportion of PSEs to supporting green operations tends to 1 at a faster rate, and the system will evolve towards an ideal state for operational management of GBs. However, when $r$ decreases to 0.5 , as $t$ changes, the system will evolve towards a state that is not conducive to operational management of GBs.

\section{Discussion}

The results of this study can be explained as follows: First, cost-benefit is one of the most important issues for companies with a profitability goal. In addition to traditional property service projects, GPM also includes energy management, water management, etc. resulting in a certain incremental cost. Therefore, PSEs need to increase their service fees before they are willing to implement GPM. However, China does not include the charging standards and related policies for GPM at present, which results the GB cannot achieve energy savings. In addition, the current GPM in China is still in the demonstration stage, mainly in Shenzhen. Most PSEs lack awareness regarding 'green service' and are not willing to actively change the traditional service model.

Secondly, GPM has significant externalities. PSEs are unable to obtain the environmental and social benefits of GPM. If the corresponding economic or other forms of compensation are given to make up for the externalities 

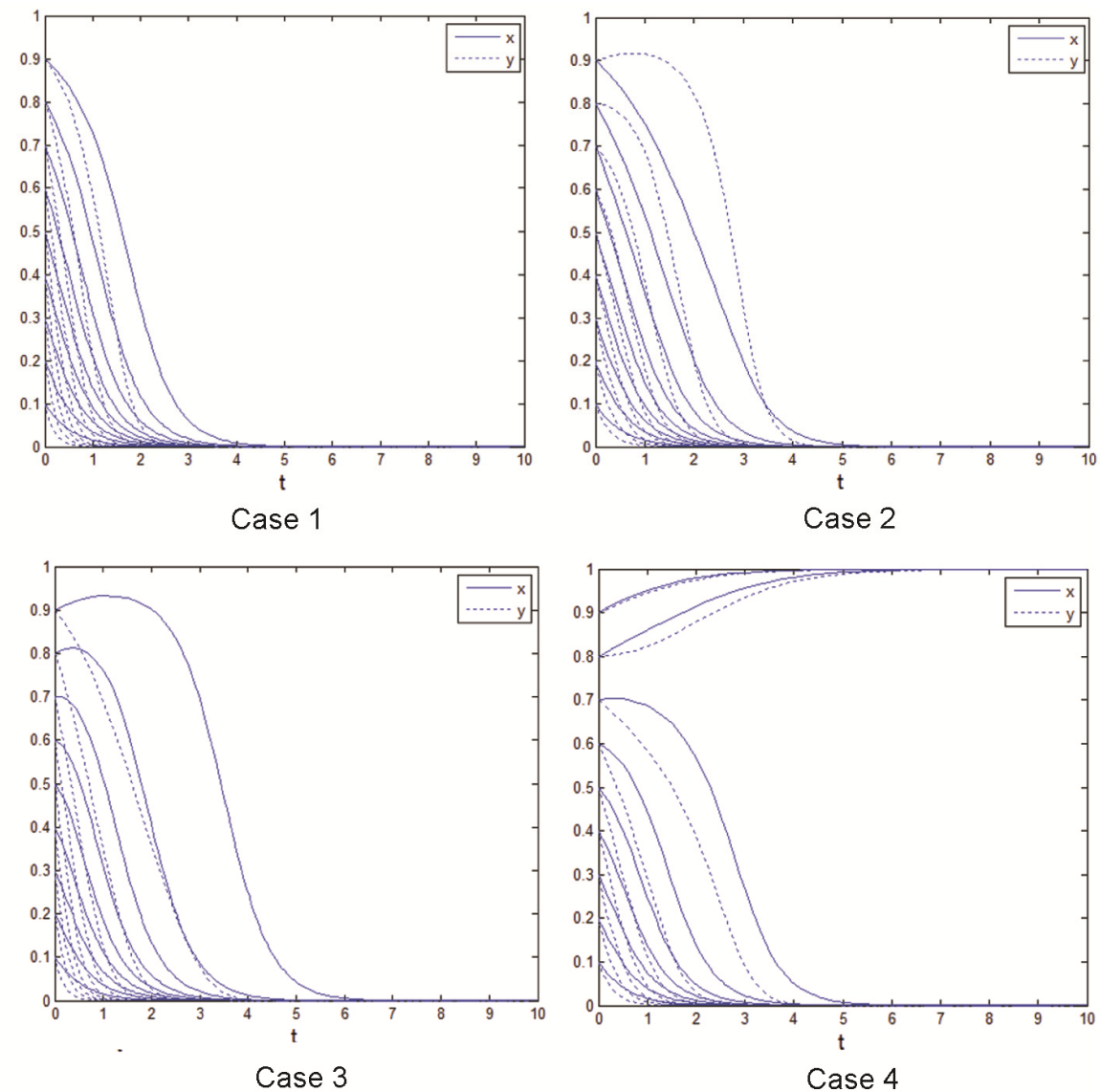

Figure 4. Equilibrium stability results under different circumstances.

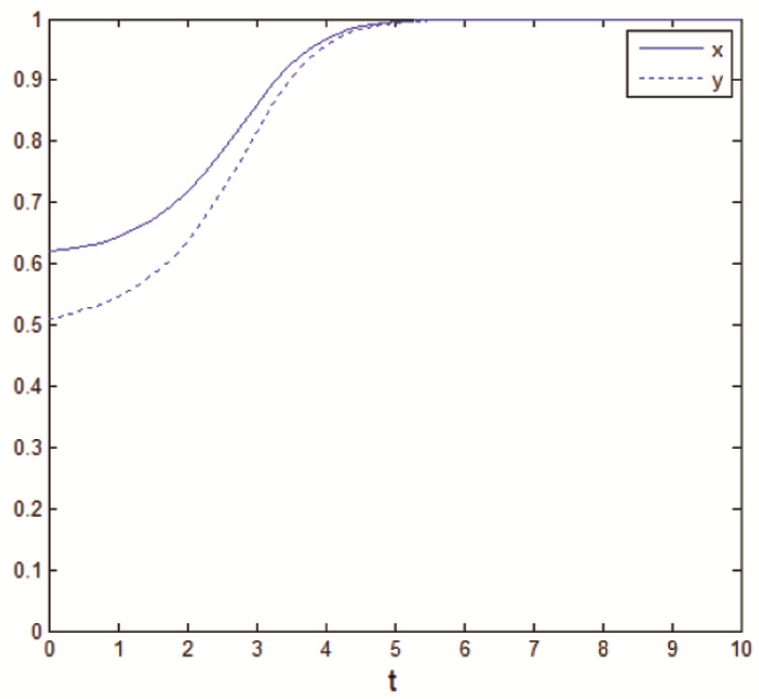

Figure 5. Evolution path diagram of the initial conditions.

generated by the GPM, the PSEs will enable to obtain certain additional benefits which can stimulate the enthusiasm of the PES to enhance the green operation capability. Meanwhile, the interests between the owners and PSEs can be coordinated.
Thirdly, PSEs have an absolute advantage in the principal-agent relationship, while the owner is at a disadvantage for they cannot directly observe the information about the PSE's ability and effort level. On the one hand, the owner can try to collect more comprehensive 

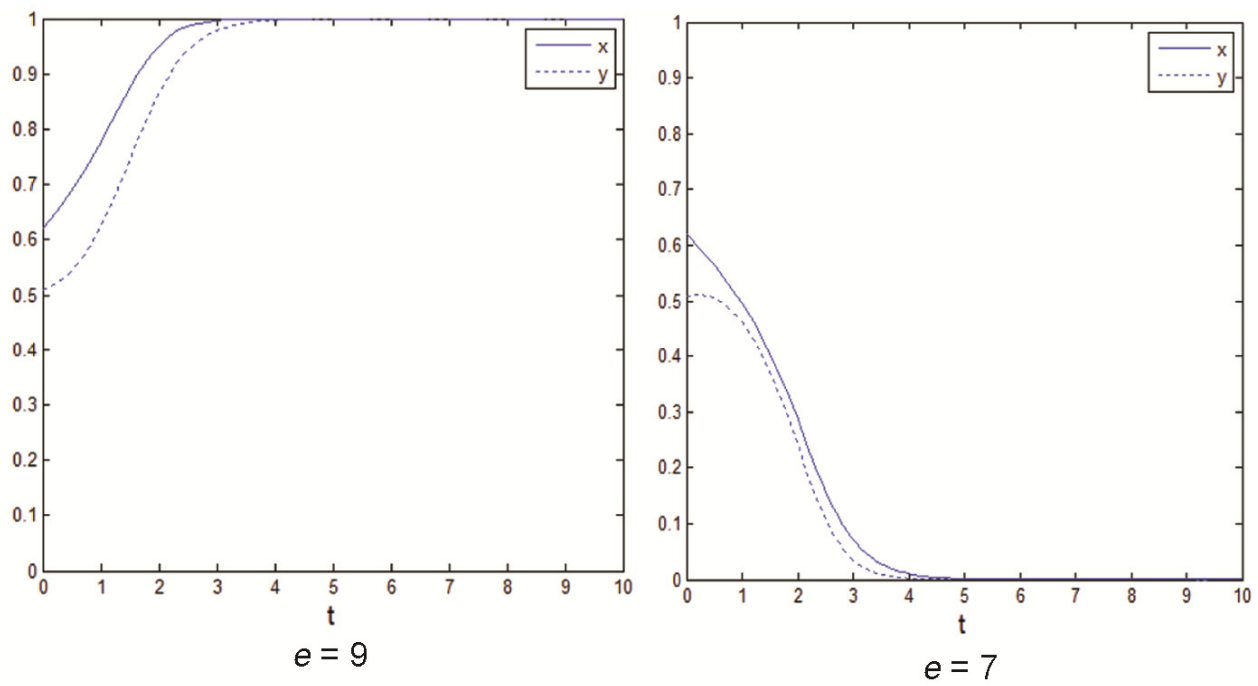

Figure 6. Evolution path diagram at different $e$ values.
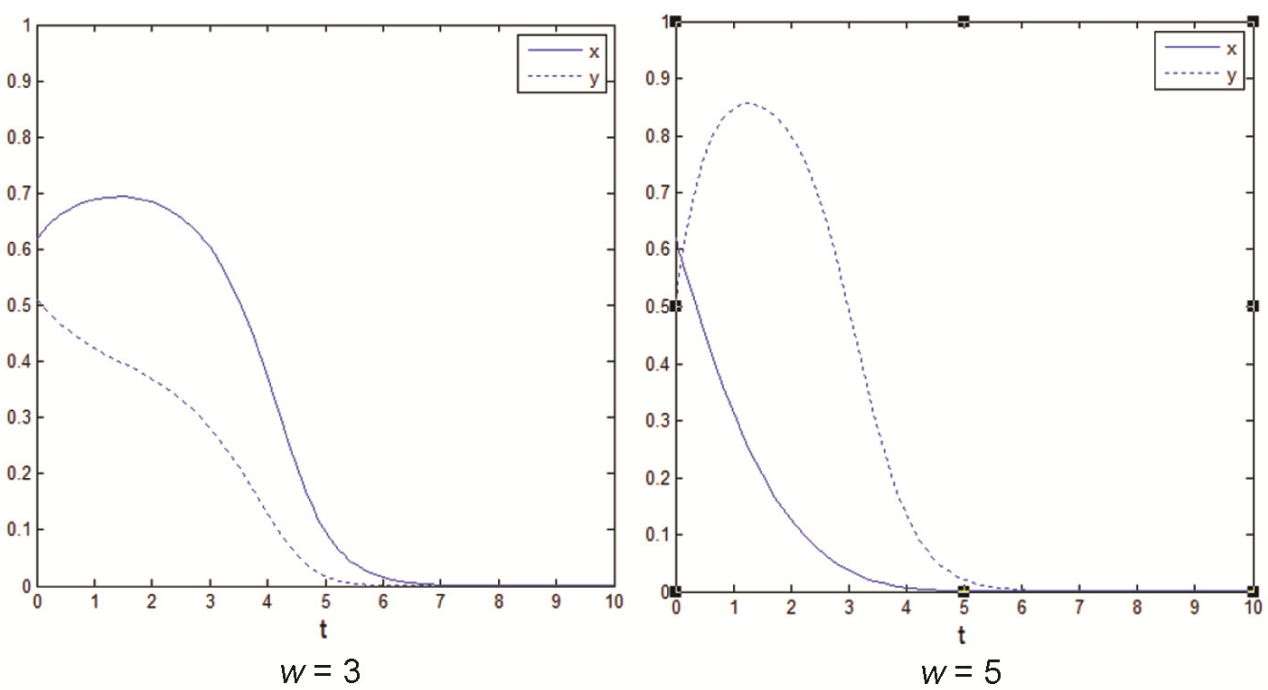

Figure 7. Evolution path diagram at different $w$ values
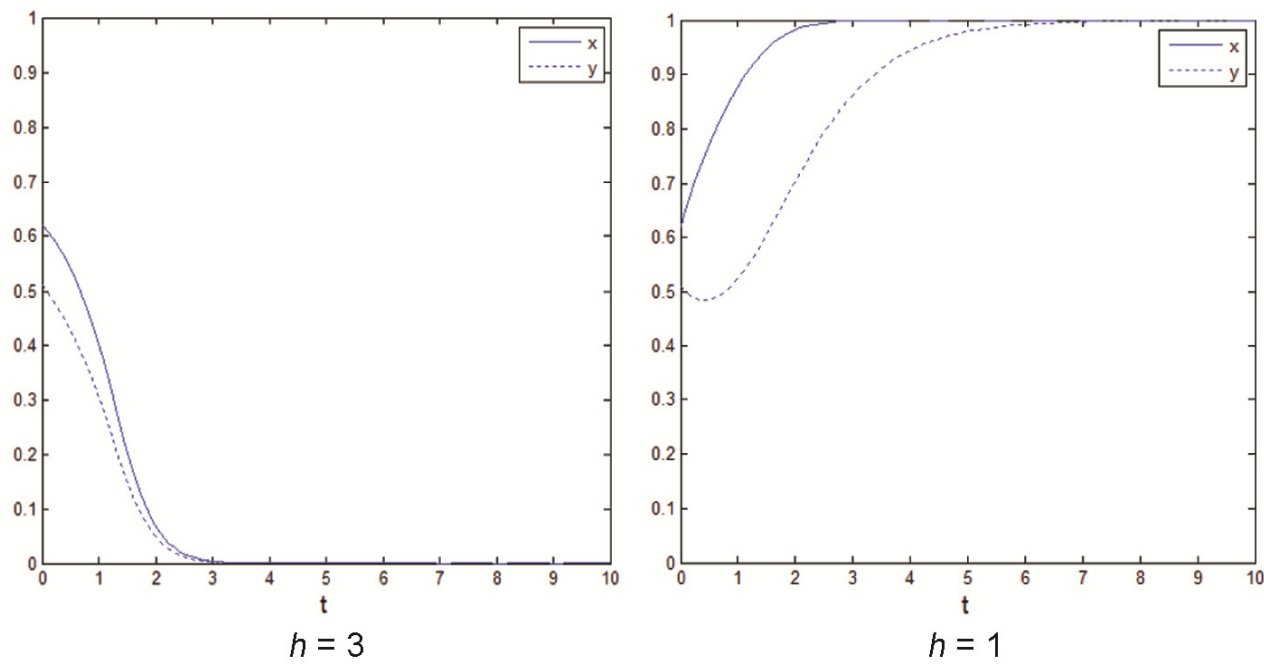

Figure 8. Evolution path diagram at different $h$ values. 

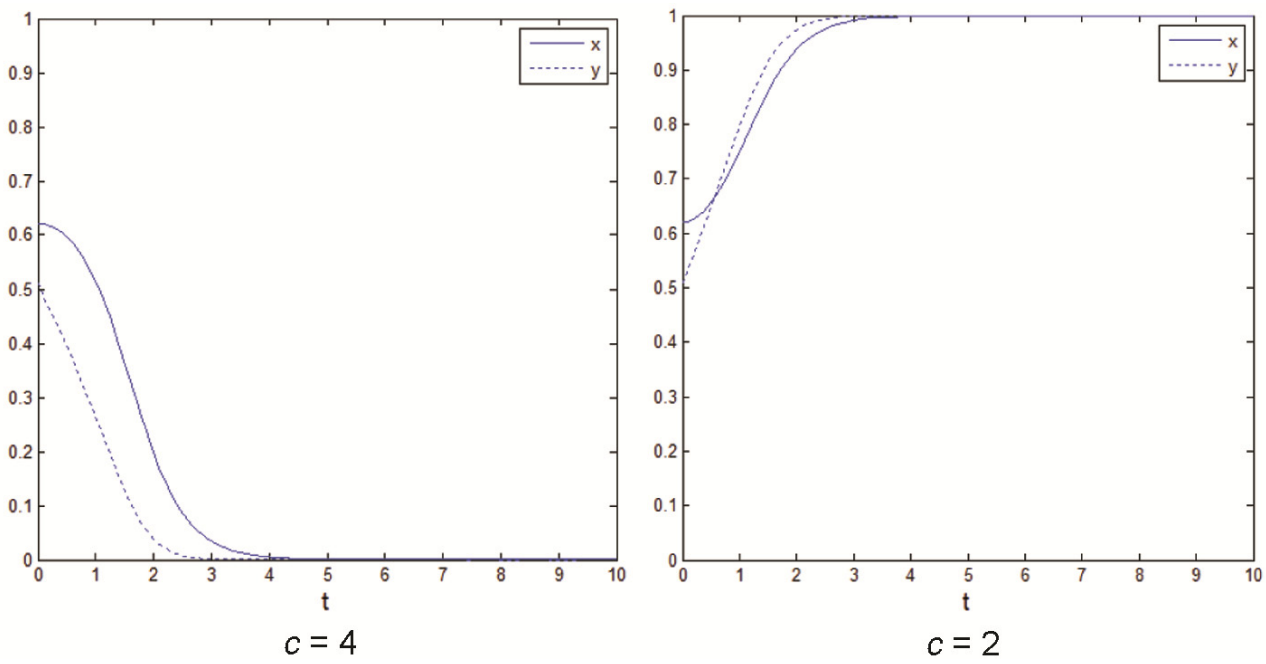

Figure 9. Evolution path diagram at different $c$ values.
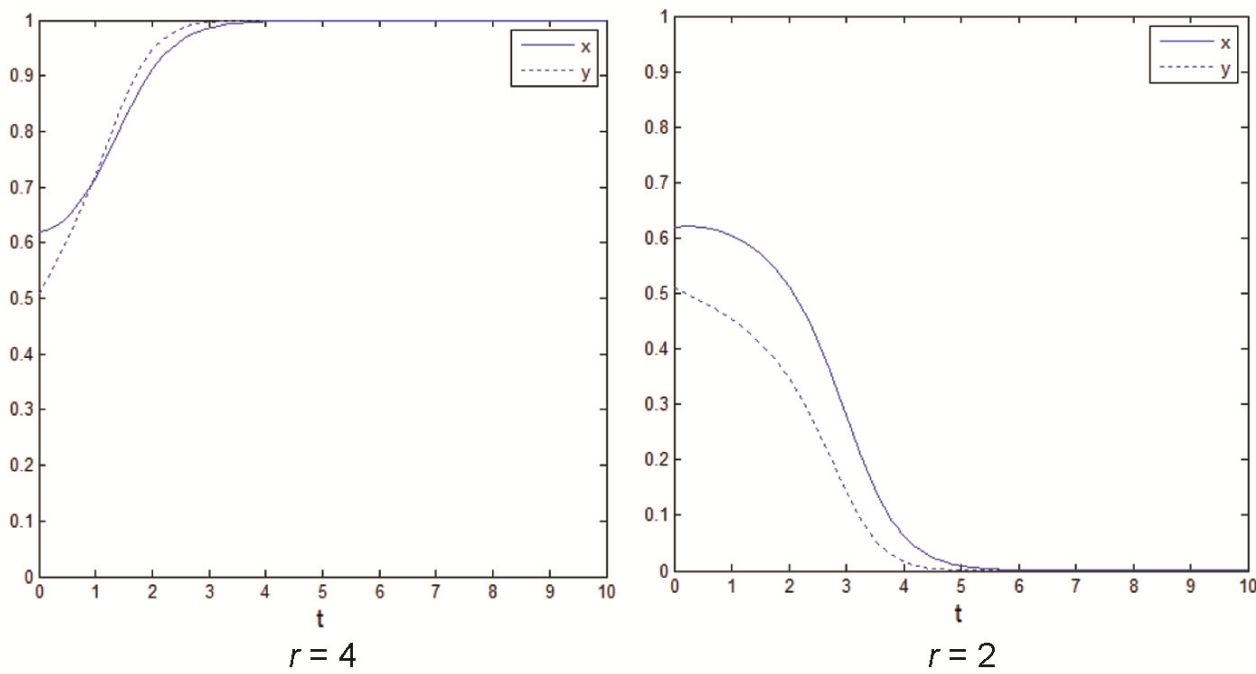

Figure 10. Evolution path diagram at different $r$ values.

information to identify the service quality of PSEs and choose a more reasonable PSE. But this will inevitably increase the transaction cost of the owners and reduce their enthusiasm to support GPM. On the other hand, due to inconsistency in the interests between owners and PSEs, moral hazard is easily generated when information is incomplete. Therefore, owners need to provide economic incentives for PSEs from an economic perspective and sign contractual contracts which allow PSEs to share energy-saving benefits.

\section{Conclusion}

The interest and conflict of interest between owners and PSEs in OS of GBs lie in the unequal distribution of operational management costs and operational benefits. In this study, we have developed an evolutionary game model of owners and PSEs. Equilibrium stability results in four cases were obtained by simulating the learning and dynamic adjustment processes of PSEs and owners. The study shows that the system will evolve towards the ideal equilibrium stability strategy only under the fourth condition. We also carried out quantitative tests for equilibrium stability results and analysed the factor that influence the evolution path. We found that improving $e$, increasing $r$, reducing $c$ and $h$, and adopting a reasonable revenue sharing method are effective ways to coordinate the interests of the owners and PSEs.

1. Li, Y. et al., Green building in China: needs great promotion. Sustain. Cities Soc., 2014, 11, 1-6.

2. Zuo, J. et al., Green building evaluation from a life-cycle perspective in Australia: a critical review. Renew. Sustain. Energ. Rev., 2017, 70, 358-368.

3. Gong, Y. et al., Life cycle assessment applied to green building certification in South Korea. Proc. Eng., 2015, 118, 1309-1313.

CURRENT SCIENCE, VOL. 117, NO. 5, 10 SEPTEMBER 2019 
4. Liao, C. Y., Tan, D. L. and Li, Y. X., Research on the application of BIM in the operation stage of green building. Appl. Mech. Mater., 2012, 5, 2111-2114.

5. Fu, H., Manogaran, G., Wu, K., Cao, M., Jiang, S. and Yang, A., Intelligent decision-making of online shopping behavior-based on internet of things. Int. J. Inf. Manage., 2019; doi:10.1016/ j.ijinfomgt.2019.03.010

6. Yu, L. et al., The practice research based on the POE system of environmental performance of green residential building. Proc. Eng., 2016, 146, 204-209.

7. Li, H., Ng, S. T. and Skitmore, M., Stakeholder impact analysis during post-occupancy evaluation of green buildings - a Chinese context. Build. Environ., 2018, 128, 89-95.

8. Shi, Q. et al., Objective conflicts in green buildings projects: a critical analysis. Build. Environ., 2016, 96, 107-117.

9. Limaei, S. M., Mixed strategy game theory, application in forest industry. For. Policy Econ., 2010, 12(7), 527-531.

10. Wang, M., Evolutionary game theory based evaluation system of green building scheme design. Cogn. Syst. Res., 2018, 52, 622 628.

11. Anna, Game analysis of the economic incentive policies of the green building demand side. J. Ecol. Econ., 2012, 2, 107-110.
12. Chen, C., Pearlmutter, D. and Schwartz, M., A game theory-based assessment of the implementation of green building in Israel. Build. Environ., 2017, 125, 122-128.

13. Liu, G. and Wei, H. Q., Study of green building operations management incentive mechanism design based on externality. J. Tianjin Chengjian Univ., 2017, 23(2), 134-138.

14. Tang, M., Sun, D. M. and Ma, S. Z., Research and analysis on operating result and carbon emissions of green building. Constr. Technol., 2012, 41(3), 30-33.

15. Huo, Y., Incentive for optimization of the green building operation under supply-side reform. Value Eng., 2017, 36(12), 1-3.

ACKNOWLEDGEMENT. We thank the Key Research and Development Plan of Shaanxi Province, China (No. 2018ZDCXL-SF-03-04) for financial support.

Received 12 November 2018; revised accepted 29 May 2019

doi: $10.18520 / \mathrm{cs} / \mathrm{v} 117 / \mathrm{i} 5 / 821-829$ 